\title{
Radiographic imaging with cosmic-ray muons
}

\section{Natural background particles could be exploited to detect concealed nuclear materials.}

D espite its enormous success, X-ray radiography ${ }^{1}$ has its limitations: an inability to penetrate dense objects, the need for multiple projections to resolve three-dimensional structure, and health risks from radiation. Here we show that natural background muons, which are generated by cosmic rays and are highly penetrating, can be used for radiographic imaging of medium-to-large, dense objects, without these limitations and with a reasonably short exposure time. This inexpensive and harmless technique may offer a useful alternative for detecting dense materials - for example, a block of uranium concealed inside a truck full of sheep.

In X-ray radiography, the intensity of an image pixel is determined by the attenuation of the incident beam caused by absorption and scattering - the maximum mean free path for photons is about $25 \mathrm{~g} \mathrm{~cm}^{-2}$ for all materials, corresponding to less than 2 $\mathrm{cm}$ of lead. For thicker objects, it is better to use a different type of radiography that is based on the interaction of charged particles with matter by multiple Coulomb scattering. The many small interactions add up to yield an angular deviation that roughly follows a gaussian distribution,

$$
\frac{d N}{d \theta_{x}}=\frac{1}{\sqrt{ } 2 \pi \theta_{0}} e^{-\frac{\theta_{x}^{2}}{2 \theta_{0}^{2}}}
$$

with the width, $\theta_{0}$, related to the scattering material through its radiation length, $L_{0}$, as follows:

$$
\theta_{0}=\frac{13.6}{\beta c p} \vee \frac{L}{L_{0}}\left[1+0.038 \ln \left(L / L_{0}\right)\right]
$$

where $p$ is the particle's momentum in $\mathrm{MeV} \mathrm{c}^{-1}$ and $\beta c$ is its velocity ${ }^{2}$. The radiation length decreases rapidly as the atomic number of a material increases, and $\theta_{0}$ increases accordingly: in a layer $10 \mathrm{~cm}$ thick, a 3-GeV muon will scatter with an angle of 2.3 milliradians in water, 11 milliradians in iron and 20 milliradians in lead. By tracking the scattering angles of individual particles, the scattering material can be mapped.

Our new technique relies on the scattering of atmospheric muons produced by primary cosmic rays. Muons are the most numerous cosmic-ray particles at sea level, moving at a rate of about $10,000 \mathrm{~m}^{-2} \mathrm{~min}^{-1}$ in horizontal detectors ${ }^{3}$. These particles are highly penetrating: a typical cosmic-ray

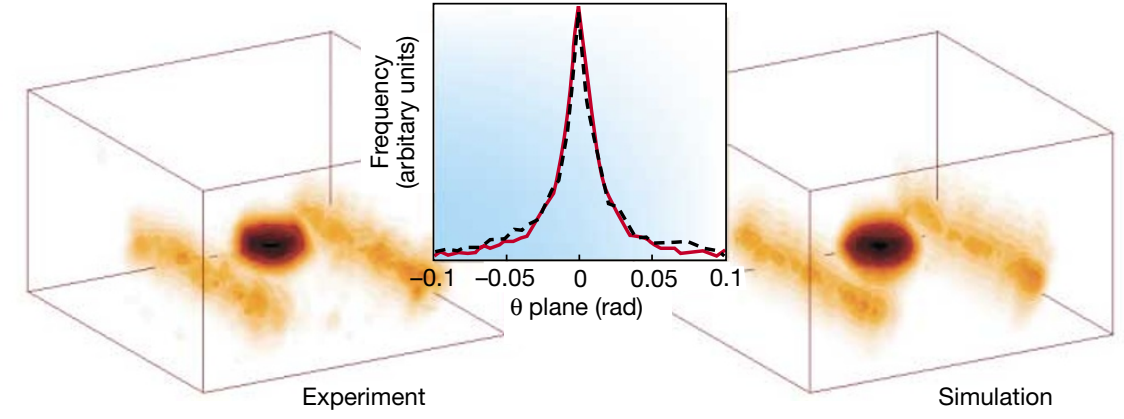

Figure 1 Radiographic imaging with muons of a test object (left) and the reconstructed image of its Monte Carlo simulation (right) The test object is a tungsten cylinder (radius, $5.5 \mathrm{~cm}$; height, $5.7 \mathrm{~cm}$ ) on a plastic $\left(35 \times 60 \times 1 \mathrm{~cm}^{3}\right)$ plate with two steel support rails. The tungsten cylinder and the iron in the rails are clearly visible in both the experiment and simulation reconstructions. Inset, the widths of the scattering distributions for tracks passing through the tungsten target are very similar for the experimental and simulated data.

muon of energy $3 \mathrm{GeV}$ will penetrate more than $1,000 \mathrm{~g} \mathrm{~cm}^{-2}(10 \mathrm{~m}$ of water, for example).

To demonstrate the concept of muon radiography, we developed a small-scale experimental system with four drift chamber detectors ${ }^{4}$ spaced $27 \mathrm{~cm}$ apart. Each detector has an active area of $60 \times 60 \mathrm{~cm}^{2}$ and records particle tracks at two positions in each of two orthogonal coordinates. The upper pair of detectors records the tracks of incident muons, and the lower pair records the scattered tracks. A tungsten cylinder was used as a test object, supported by a plastic plate and steel support beams. The tungsten is clearly visible in the reconstructed image, and the steel support beams are also evident (Fig. 1, left).

We also developed a Monte Carlo simulation code that generated cosmic-ray muons and propagated them through a test volume. The reconstructed images are indistinguishable from those obtained experimentally (Fig. 1), and the scatter angles of the simulated muons from the different materials (tungsten, lexan and steel) are consistent with the measured angles.

Simulation of larger, more complex objects demonstrates that we can reliably detect a $10 \times 10 \times 10 \mathrm{~cm}^{3}$ uranium object inside a large metal container full of sheep in $1 \mathrm{~min}$ of exposure. We conclude that cosmic-ray muons show promise as an inexpensive, harmless probe for radiography of medium-to-large objects, such as commercial trucks, passenger cars or sea containers. Our experimental results and simulations demonstrate the ability to reconstruct complex objects and to detect dense material of high atomic number hidden in a much larger volume of material of low atomic number, using only the natural flux of muons. This method is suitable for a range of practical applications in which radiography of dense objects with low radiation dose is required - for example, in surveillance for cross-border transport of nuclear materials.

Konstantin N. Borozdin, Gary E. Hogan,

Christopher Morris, William C.

Priedhorsky, Alexander Saunders,

Larry J. Schultz, Margaret E. Teasdale

Los Alamos National Laboratory, Los Alamos,

New Mexico 87545, USA

e-mail:kbor@lanl.gov

1. Roentgen, W. C. Nature 53, 274 (1896)

2. Hagiwara, K. et al. Phys. Rev. D 66, 01001 (2002).

3. Grieder, P.K.F. Cosmic Rays at Earth (Elsevier Science, Amsterdam, 2001)

4. Atencio, L. G., Amann, J. F., Boudrie, R. L. \& Morris, C. L. Nucl. Instr. Methods Phys. Res. 187, 381-386 (1981).

Competing financial interests: declared none.

\section{Palaeo-oceanography}

\section{Deepwater variability in the Holocene epoch}

he conversion of surface water to deep water in the North Atlantic results in the release of heat from the ocean to the atmosphere, which may have amplified millennial-scale climate variability during glacial times ${ }^{1}$ and could even have contributed to the past 11,700 years of relatively mild climate (known as the Holocene epoch $)^{2-4}$. Here we investigate changes in the carbon-isotope composition of benthic foraminifera throughout the Holocene and find that deep-water production varied on a centennial-millennial timescale. These variations may be linked to surface and atmospheric events that hint at a contribution to climate change over this period. 


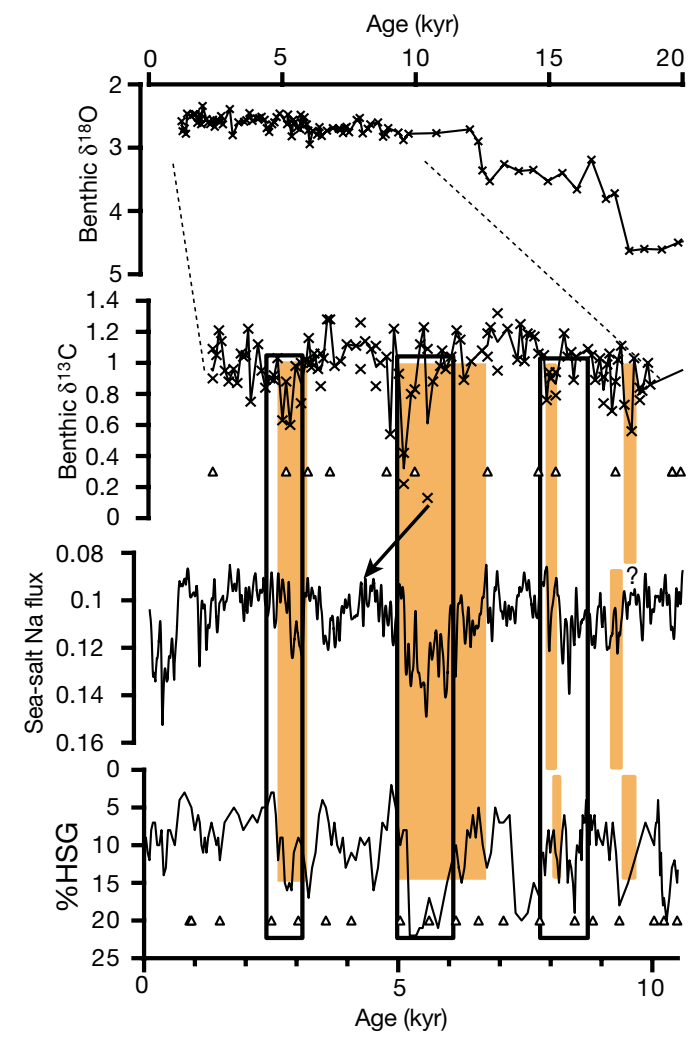

Figure 1 Holocene climate records, top to bottom: benthic $\delta^{18} 0$ and $\delta^{13} \mathrm{C}$ (North Atlantic deepwater contribution) records from site 980 (ref. 12, and see supplementary information); GISP2 sea-salt sodium flux ${ }^{8}$ (increasing, calm to windier), and percentage of haematite-stained grains (\%HSG) in core V29-191 (ref. 2). Triangles, dated levels in sediment cores; arrow marks the onset and intensificatoin of the 5kyr event. Accelerator mass-spectrometer radiocarbon dates converted to calendar age ${ }^{13}$ from site 980 provided the chronology for the Holocene (see supplementary information). Accumulation rates average about $25 \mathrm{~cm} \mathrm{kyr}^{-1}$ in the Holocene, roughly double that in nearby core V29-191. The average interval between samples is about 100 yr from 9.7 to $1.2 \mathrm{kyr}$. Shading shows low- $\delta{ }^{13} \mathrm{C}$ events (see text) and possible correlative events in the other records; a more tenuous correlation is denoted by the question mark. Rectangles, extreme winter-like conditions deduced from statistical analysis of the full set of palaeochemical indicators from GISP2 (ref. 8).

We used an established deepwater proxy parameter - the carbon-isotope composition $\left(\delta^{13} \mathrm{C}\right)$ of the epifaunal benthic foraminifera Cibicidoides wuellerstorf $i^{5,6}$ to evaluate Holocene deepwater variability. Variations in the $\delta^{13} \mathrm{C}$ of the total amount of $\mathrm{CO}_{2}$ in bottom waters, which is accurately recorded by $C$. wuellerstorfi, can be used to monitor variations in the contribution of high- $\delta{ }^{13} \mathrm{C}$ North Atlantic Deep Water (NADW) relative to low $-\delta^{13} \mathrm{C}$ Southern Ocean Water (SOW) to a site. We worked on sediment from Ocean Drilling Project site $980\left(55^{\circ} \mathrm{N}, 15^{\circ} \mathrm{W}\right.$; depth, $\left.2,179 \mathrm{~m}\right)$, on the Feni Drift in the subpolar northeastern Atlantic.

Holocene $\delta^{13} \mathrm{C}$ values (Fig. 1, and see supplementary information) show marked millennial oscillations around the modern value of about $1 \%$ o (ref. 7 ). Values range from a high of $1.3 \%$ to values lower than $0.6 \%$, indicating times of enhanced and reduced NADW contribution, respectively. The largest reductions in the relative NADW contribution occurred around 9,300 years ago (9.3 kyr), and 8.0, 5.0 and $2.8 \mathrm{kyr}$ ago. Smaller events occurred more frequently.

The most pronounced feature of the Holocene is a trend of decreasing relative NADW contribution that began at about $6.5 \mathrm{kyr}$ and culminated with a minimum at around 5 kyr. Statistical analysis of the full set of chemical records from the Greenland Ice Sheet Project 2 (GISP2) ice core indicates that meteorological conditions from
6.1 to $5.0 \mathrm{kyr}$ were especially winter-like (characterized by an expanded polar vortex) at high latitudes; this is consistent with high values of sea-salt sodium (Fig. 1) during this millennial-scale event ${ }^{8}$.

A high relative abundance of haematitestained grains in a nearby core indicates a large proportion of cold, fresh, ice-bearing surface water from north of Iceland ${ }^{2}$. All three records indicate that the 5-kyr event was the most severe climate event of our Holocene study interval. Apparently contemporary variations in benthic $\delta^{13} \mathrm{C}$ and sea-salt sodium levels indicate that linked, century-scale variability may have occurred during this event.

The GISP2 palaeochemical records indicate enhanced winter-like conditions from 2.4 to $3.1 \mathrm{kyr}$, and from 8.8 to $7.8 \mathrm{kyr}$, although to a lesser extent than in the 5-kyr event $^{8}$. The more recent of these cold intervals corresponds precisely to the reduced NADW contribution at around $2.8 \mathrm{kyr}$. The 8 -kyr $\delta^{13} \mathrm{C}$ minimum occurs late in the 8.8-7.8-kyr cold interval, but corresponds to a brief maximum in sea-salt sodium. The age of the 9.3-kyr event is poorly constrained, but it may correspond to one of several younger or older maxima in sea-salt sodium flux. Three of the four low $-\delta^{13} \mathrm{C}$ events correspond to maxima in the percentage of haematite-stained grains (increased incursion of rock-bearing ice from north of Iceland), suggesting a possible surface-deepwater linkage.
Prior geochemical evidence for deepwater reduction in the Holocene was limited to events outside our study interval. Low $\delta^{13} \mathrm{C}$ values during the Little Ice Age $\mathrm{A}^{4}$, the most recent of the Holocene millennial cold events ${ }^{8}$, and during an early Holocene event at $\sim 10.3 \mathrm{kyr}$ (ref. 9) hint at a linkage between millennial climate and deep water. Downcore variations in sedimentological indices also indicate this possibility ${ }^{10,11}$.

The new North Atlantic benthic $\delta^{13} \mathrm{C}$ record unambiguously demonstrates that NADW varied on centennial-millennial time scales during the Holocene. The most significant Holocene event of reduced NADW contribution, which occurred at $5 \mathrm{kyr}$, was correlated to winter-like atmospheric conditions at high northern latitudes ${ }^{8}$, and to incursion of sea ice from north of Iceland ${ }^{2}$. Similarly, a more recent cold event (at 3.1-2.4 kyr) was associated with a reduced NADW contribution.

Evidence for a climate-deepwater linkage during the earlier events is weaker, and may indicate an increasing sensitivity of deepwater to surface forcing from the Early to the Late Holocene. Further welldated deepwater proxy records are needed to test this possibility.

Our study raises other important issues that can be addressed through data collection and modelling. The amplitude of the largest Holocene $\delta^{13} \mathrm{C}$ fluctuation near $5 \mathrm{kyr}$, for example, is similar to those of $\delta^{13} \mathrm{C}$ oscillations during earlier glacial and deglacial events ${ }^{12}$. This suggests that significant variations in the relative NADW contribution can occur in the absence of forcing by large ice sheets, and that NADW may be more sensitive to surface forcing than was previously imagined.

Delia W. Oppo*, Jerry F. McManus*, James L. Cullen $\dagger$

${ }^{\star}$ Department of Geology and Geophysics, Woods Hole Oceanographic Institution, Woods Hole,

Massachusetts 02540, USA

e-mail:doppo@whoi.edu

$\dagger$ Department of Geological Sciences, Salem State

College, Salem, Massachusetts 01970, USA

1. Broecker, W. S., Bond, G. \& Klas, M. Paleoceanography 5 , 469-477 (1990).

2. Bond, G. C. et al. Science 294, 2130-2136 (2001).

3. Alley, R. B. et al. Geology 25, 483-486 (1997)

4. Keigwin, L. D. \& Boyle, E. A. Proc. Natl Acad. Sci. USA 97, 1343-1346 (2000).

5. Curry, W. B., Duplessy, J. C., Labeyrie, L. D. \& Shackleton, N. J. Paleoceanography 3, 317-341 (1988).

6. Duplessy, J. C. et al. Paleoceanography 3, 343-360 (1988).

7. Kroopnick, P. M. Deep-Sea Res. 32, 57-84 (1985).

8. O'Brien, S. R. et al. Science 270, 1962-1964 (1995).

9. Bond, G. C. et al. Science 278, 1257-1266 (1997).

10. Bianchi, G. G. \& McCave, I. N. Nature 397, 515-517 (1999).

11. Chapman, M. R. \& Shackleton, N. J. Holocene 10, 287-291 (2000).

12. McManus, J. F., Oppo, D. W. \& Cullen, J. L. Science 283, 971-975 (1999).

13. Stuiver, M. \& Braziunas, T. F. Radiocarbon 35, 137-189 (1993). Supplementary information accompanies this communication on Nature's website.

Competing financial interests: declared none. 


\section{Ecology}

\section{Hunting and fox numbers in the United Kingdom}

- he potential impact of fox-hunting ban in Britain is a contentious issue $e^{1}$ that has been explored by Baker et al. ${ }^{2}$. They conclude that a suspension of lowland foxhunting for nine months during 2001 made no difference to fox density in certain areas. We are not confident, however, that their analysis supports their conclusions - their study does not consider statistical power or account sufficiently for regional variation, and also uses an inappropriate statistic.

An analysis of statistical power is necessary to demonstrate the probability of detecting an increase in fox density of $10 \%, 20 \%, 30 \%$, and so on, given the sample sizes and variability of the data. It seems likely that the power of Baker and colleagues' study to detect plausible effect sizes was low, whether by the analysis published or by any other test: the proper conclusion should be 'no evidence', rather than 'evidence of no effect'.

Moreover, a constant of 1.0 used (twice) in calculating the relative change in faecal abundance, $R^{\prime}$, is large by comparison with average scat density, with the result that $R^{\prime}$ is higher where faecal density before the hunting ban (imposed during the outbreak of foot-and-mouth disease (FMD)) is higher. For the same proportional increase in faecal density, $R^{\prime}$ takes lower values for a low pre-FMD density than for a high pre-FMD density. For example, a 50\% increase from a pre-FMD density of 5 faeces per $\mathrm{km}^{2}$ gives $R^{\prime}=0.008$, whereas the same $50 \%$ increase from a pre-FMD density of 100 per $\mathrm{km}^{2}$ gives $R^{\prime}=0.085$ (we have used $\log _{10}$; average faecal density was 50 faeces per $\mathrm{km}^{2}$ (ref. 3) and the average transect length was $6.9 \mathrm{~km}$ (ref. 2)).

If, as hypothesized by Baker et al., preFMD fox density had been suppressed in hunted squares, initial faecal density would be low in those squares, but would be predicted to increase during the suspension of hunting. Because of the low initial density, $R^{\prime}$ would take low values in those squares. In an analysis of all squares, this would tend to mask differences between squares that show an effect and those that show no effect (for no change, $R^{\prime}=0$ ). The consequences of this could influence the conclusions of Baker et al.

Finally, an earlier study contrasting three large regions of England and Wales concluded that hunting with dogs was the major part of an effective cull in one region, but not in the other two ${ }^{4}$. It is therefore appropriate to test for regional variation in the impact of hunting pressure, requiring that an interaction term be explicitly modelled. The upland regions where hunting with dogs is more likely to suppress fox densities were not represented in Baker and colleagues' study.

Only when these concerns are addressed can we concur that Baker et al. have clarified the effect of a hunting ban on fox populations. Nicholas J. Aebischer ${ }^{\star}$, Sandra E. Baker $\dagger$, Paul J. Johnson $\dagger$, David W. Macdonald $\dagger$, Jonathan C. Reynolds*

* The Game Conservancy Trust, Fordingbridge, Hampshire SP6 1EF, UK

$\dagger$ The Wildlife Conservation Research Unit, Department of Zoology, University of Oxford, South Parks Road, Oxford OX1 3PS, UK

1. Macdonald, D. W. et al. Managing British Mammals: Case Studies from the Hunting Debate (Wildlife Conserv. Res. Unit, Oxford, 2000).

2. Baker, P. J., Harris, S. \& Webbon, C. C. Nature 419, 34 (2002).

. The Mammal Society (www.mammal.org.uk).

4. Heydon, M. J. \& Reynolds, J. C. J. Zool. 251, 265-276 (2000)

Baker et al. reply - Although there is a tendency for our transformation to result in low $R^{\prime}$ values for those squares where initial faecal density was low, this does not affect our results. First, this 'bias' is equivalent to a 1.2 -fold difference in $R^{\prime}$ for a 20 -fold difference in initial faecal density ${ }^{1}$. Second, there was no difference between 'hunted' and 'nonhunted' squares with respect to initial faecal density, direction or magnitude of change in faecal density ${ }^{1}$. The starting conditions and pattern of change were therefore the same for hunted and non-hunted squares, and the reservations of Aebischer et al. about the transformation are unwarranted.

Our key hypothesis was that hunting with hounds (hereafter termed 'hunting') is additive to other culling practices ${ }^{2}$. Consequently, the absence of hunting during FMD would be expected to result in increased fox abundance in areas that were previously hunted. The most parsimonious way to test this hypothesis, and to remove any possibility of a transformation effect, is by using a signs test ${ }^{3}$ - this compares the number of squares in which scat density increased with the number in which it decreased, assuming a 50\% chance of either event. This negates the need for a regional approach - if hunting is additive, a change of any magnitude in any region would be detected using our paired samples ${ }^{2}$.

To determine the power of this approach, we need the $\alpha$-error rate $(\alpha=0.05)$, the sample size $(n=157$; squares with no change are excluded) and the size of the likely effect ${ }^{3}$. In previous studies ${ }^{4-6}$, the impact of hunting in Britain (total area, $230,367 \mathrm{~km}^{2}$ ) has been estimated by extrapolating kill rates per unit area to the total area covered by packs of foxhounds $\left(145,000 \mathrm{~km}^{2}\right)$. The proportion of land in Britain that is hunted is therefore 0.63 .

Statistical power is the probability of correctly rejecting a false null hypothesis. For a two-tailed test, the minimum effect size $(g)$ is given by: $0.63-0.50=0.13$; the statistical power that corresponds to $\alpha=0.05, n=157$ and $g=0.13$ is about 0.950 (ref. 3 ). We therefore had a $95 \%$ chance of correctly rejecting a false null hypothesis, for a roughly $13 \%$ deviation from a probability that faecal density would increase (or decrease) in $50 \%$ of squares. The actual results showed no change $(P=0.474)$. The observed pattern of variation in faecal density is therefore not consistent with hunting mortality being additive. Furthermore, the small absolute changes in faecal density ${ }^{2}$ indicate minor changes in fox density. We reiterate that these results support the Committee of Inquiry into Hunting with Dogs $^{6}$, which concluded that a permanent ban on hunting is unlikely to result in a dramatic increase in fox numbers.

Philip J. Baker, Stephen Harris, Charlotte C. Webbon

School of Biological Sciences, University of Bristol, Woodland Road, Bristol BS8 1UG, UK

e-mail:s.harris@bristol.ac.uk

\footnotetext{
1. The Mammal Society (www.mammal.org.uk).

2. Baker, P. J., Harris, S. \& Webbon, C. C. Nature 419, 34 (2002).

3. Cohen, J. Statistical Power Analysis for the Behavioural Sciences (Academic, London, 1977).

4. Macdonald, D. W. \& Johnson, P. J. in The Exploitation of Mammal Populations (eds Taylor, V. J. \& Dunstone, N.) 160-207 (Chapman \& Hall, London, 1996).

5. Macdonald, D. W. et al. Management and Control of Populations of Foxes, Deer, Hares and Mink in England and Wales, and the Impact of Hunting with Dogs (Report to Lord Burns' Inquiry into Hunting with Dogs, 2000).

6. Burns, L., Edwards, V., Marsh, J., Soulsby, L. \& Winter, M Report of the Committee of Inquiry into Hunting with Dogs in England and Wales (Stationery Office, London, 2000).
}

\section{correction}

Deepwater variability in the Holocene epoch

D. W. Oppo, J. F. McManus, J. L. Cullen

Nature 422, 277-278 (2003)

In Fig. 1, the arrow marking the onset and intensification of the 5-kyr event was positioned incorrectly. The correct version of the figure is shown here.

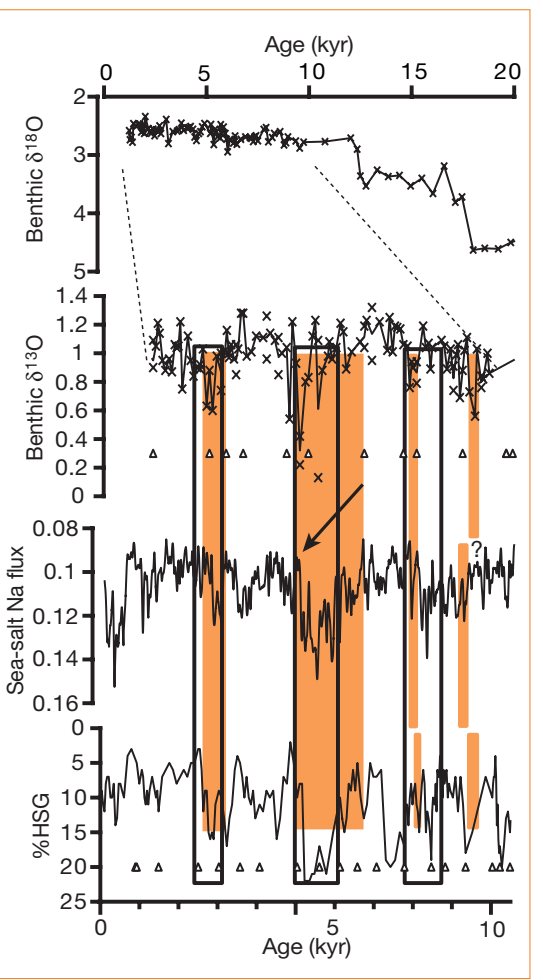

